\title{
The Impact Damage and Medium Leakage of CFRP Laminates with Different Thickness
}

\author{
Aying Zhang ${ }^{1,2, *}$, Dongxing Zhang ${ }^{2}$, Mingzhe $\mathrm{Qu}^{1}, \mathrm{Kai}_{\mathrm{Yu}}^{2}$ \\ ${ }^{1}$ Harbin University, Harbin 150086, People's Republic of China \\ ${ }^{2}$ Harbin Institute of Technology, Harbin 150001, People's Republic of China
}

\begin{abstract}
The effects of thickness and impact energy on the impact damage and the medium leakage of CFRP laminates were studied in this paper. Impact tests for the CFRP laminates with the size of $600 \mathrm{~mm} \times 700 \mathrm{~mm}$ with five different thicknesses were subjected to the impact energy levels from $5 \mathrm{~J}$ to $65 \mathrm{~J}$. The matrix length was investigated according to different energy levels and different thicknesses. The experimental results reveal that the crack length increases linearly with the increasing impact energy. For the same impact energy, the crack lengths decrease with the increasing specimen thickness. The impact energy initial value of medium leakage increases with the increasing thickness. The impact energy initial value of aviation kerosene leakage is about 6.7416 times of the thicknesses of laminates, which is coordinate with the experience value of 6.75 times.
\end{abstract}

\section{Introduction}

The high specific strength, stiffness, and good chemical resistance of carbon fiber reinforced polymer matrix composites (CFRP) make them attractive for applications from sports equipment to aerospace structures [1-4]. However, CFRP laminates are relatively brittle under the impact loading, which limit its wide applications [5-7]. Compared to other composite materials used in the aerospace field, laminated composites have excellent inplane properties but are subjected to barely visible impact damage (BVID) by out-of-plane impact loading [8-12]. CFRP laminates is susceptibility to the impact damage because of their weak interfaces, and even minor damage can cause a considerable reduction in the structural integrity [13-15]. The stiffness and the strength may be significantly reduced by the impact damage, which may result in premature structural failure [16-19].

The objective of this research was to evaluate the impact damage and medium leakage of CFRP laminates of five different thicknesses were subjected to impact loading at the energy levels from $5 \mathrm{~J}$ to $65 \mathrm{~J}$ at the room temperature. After the impact tests had concluded, the impacted specimens are subjected to additional tensile testing. The medium leakage tests under the tensile loading after impact were carried out at constant temperature and pressure.

\section{Experimental}

\subsection{Materials}

The laminates used in this study are prepregs of T800 carbon fiber/epoxy X850 (fiber volume fraction of $65 \%$ $\pm 2 \%$ ) supplied by Hexcel.

The impact damage of CFRP laminates was investigated as a function of the five stacking sequences, as shown in Table 1.
Table 1. Stacking sequences of CFRP laminates.

\begin{tabular}{|c|c|}
\hline Specimen type & Stacking sequences \\
\hline I & {$[+45 /-45 / 0 / 90 / 0 /-45 / 0 /+45]_{\mathrm{s}}$} \\
\hline II & {$[+45 /-45 / 0 / 90 / 0 /-45 / 0 / 0 / 0 /+45]_{\mathrm{s}}$} \\
\hline III & $\begin{array}{l}{[+45 /-45 / 0 / 90 / 0 /-45 / 0 / 90 / 0 /+45 / 0 / 0 /-} \\
45 / 0 /+45]_{\mathrm{s}}\end{array}$ \\
\hline IV & $\begin{array}{l}{[+45 /-45 / 0 / 90 / 0 /-} \\
45 / 0 / 0 /+45 / 0 / 90 / 0 /+45 / 0 /- \\
45 / 0 /+45 / 0 / 0 /-45]_{\mathrm{s}}\end{array}$ \\
\hline V & $\begin{array}{l}{[+45 /-45 / 0 /-45 / 90 /+45 / 0 / 0 /-} \\
45 / 0 / 90 / 0 /+45 / 0 / 90 / 0 /+45 / 0 / 0 /-45 \\
/ 0 /+45 / 0 / 0 /-45]_{\mathrm{s}}\end{array}$ \\
\hline
\end{tabular}

The plies and thickness of CFRP laminates are shown in Table 2. The specimens were cut from the woven laminates with the size of $600 \mathrm{~mm} \times 700 \mathrm{~mm}$. The laminates were constructed by hand lay-up and cured in an autoclave system.

Table 2. The plies and thickness of CFRP laminates.

\begin{tabular}{|c|c|c|}
\hline Specimen type & Plies & Thickness (mm) \\
\hline I & 16 & 3.04 \\
\hline II & 20 & 3.80 \\
\hline III & 30 & 5.70 \\
\hline IV & 40 & 7.60 \\
\hline V & 50 & 9.50 \\
\hline
\end{tabular}




\subsection{Impact test}

The DYNATUP model JLW-100 impact testing machine was used to conduct the impact tests. By varying the crosshead mass and the height of release, it was possible to reach the maximum impact energy of $200 \mathrm{~J}$. Impact was given to the specimen by the drop-weight with a hemispherical impactor of $12.7 \mathrm{~mm}$ in diameter. For this investigation, the total mass of the impactor was $5 \mathrm{~kg}$. The kinetic energy is available by varying the height of release. All the impact tests were carried out at the room temperature.

As shown in Table 3, the impact energy increase from the initial impact energy $(5 \mathrm{~J})$ to the maximum impact energy and the increment is $5 \mathrm{~J}$.

Table 3. The impact energy of CFRP laminates (J).

\begin{tabular}{|c|c|c|c|c|}
\hline \multicolumn{5}{|c|}{ Specimen type } \\
\hline I & II & III & IV & V \\
\hline 5 & 5 & 5 & 5 & 5 \\
\hline 10 & 10 & 10 & 10 & 10 \\
\hline 15 & 15 & 15 & 15 & 15 \\
\hline 20 & 20 & 20 & 20 & 20 \\
\hline 25 & 25 & 25 & 25 & 25 \\
\hline- & 30 & 30 & 30 & 30 \\
\hline- & 35 & 35 & 35 & 35 \\
\hline- & - & 40 & 40 & 40 \\
\hline- & - & - & 45 & 45 \\
\hline- & - & - & 50 & 55 \\
\hline- & - & - & - & 60 \\
\hline- & - & - & - & 65 \\
\hline
\end{tabular}

\subsection{Impact damage test}

The calipers with testing accuracy of $0.02 \mathrm{~mm}$ were used to measure the cracks length of the composite laminates. Super ultrasonic C-scan (RapidScan 2/3D) was used to measure the delamination.

\subsection{Tensile and leakage test}

The impacted specimens are subjected to the additional tensile testing after being impacted, which were conducted by two screw jacks (QL100). The tensile loading kept constant when the tensile strain being $4000 \mu \varepsilon$. The aviation fuel leakage tests were carried out at $55^{\circ} \mathrm{C}$.

\section{Results and discussion}

\subsection{Impact cracks length assessment}

Figure 1 shows that the crack lengths increase linearly with the increasing impact energy. After performing the impact test at the room temperature, the damaged areas were visually inspected on the impacted and back surfaces.

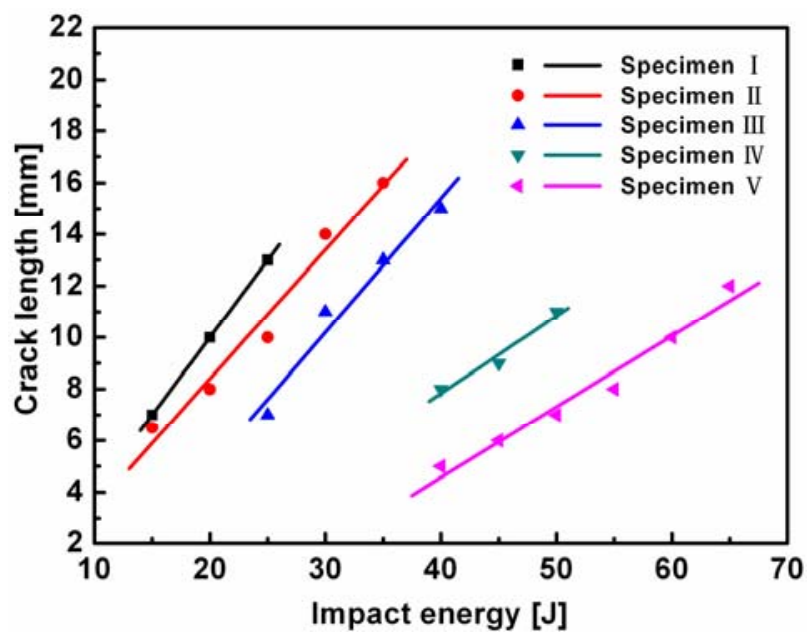

Figure 1. The crack length of CFRP laminates with different thickness.

No apparent damage was observed on either side at the impact energy of $5 \mathrm{~J}$. For the same impact energy, the crack lengths decrease with increasing of the thickness of specimens.

\subsection{Impact damage test assessment}

Figure 2 to Figure 7 shows the experimental results of the crater depth with the ultrasonic scan. As shown in Figure 2 and Figure 3, the crater depth of the same specimen increase as the impact energy increase.

Figure 3, Figure 4, Figure 6 and Figure 7shows that the impact damage is severer as the thickness of the specimen decrease at the same impact energy.

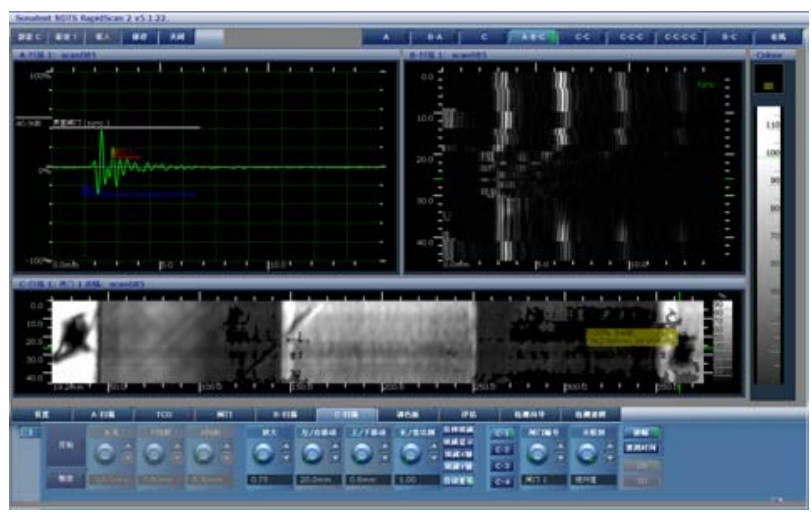

Figure 2. The crater depth of specimen I at the energy of $20 \mathrm{~J}$. 


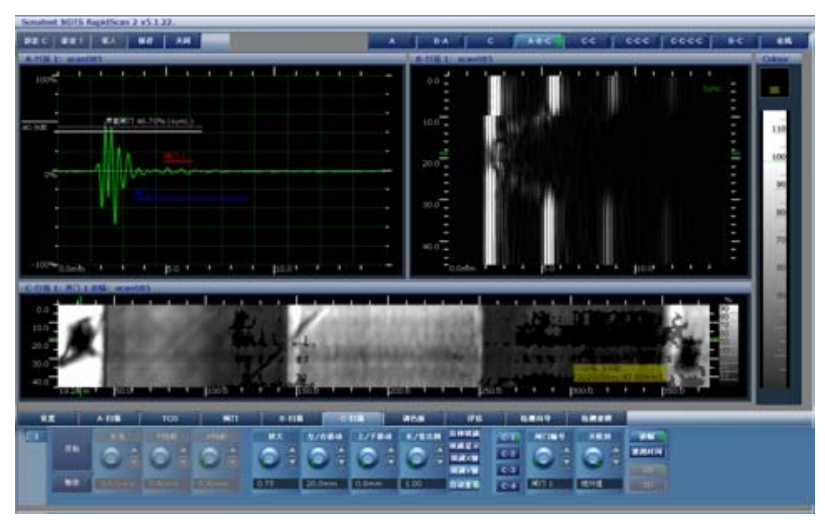

Figure 3. The crater depth of specimen $\mathrm{I}$ at the energy of $25 \mathrm{~J}$.

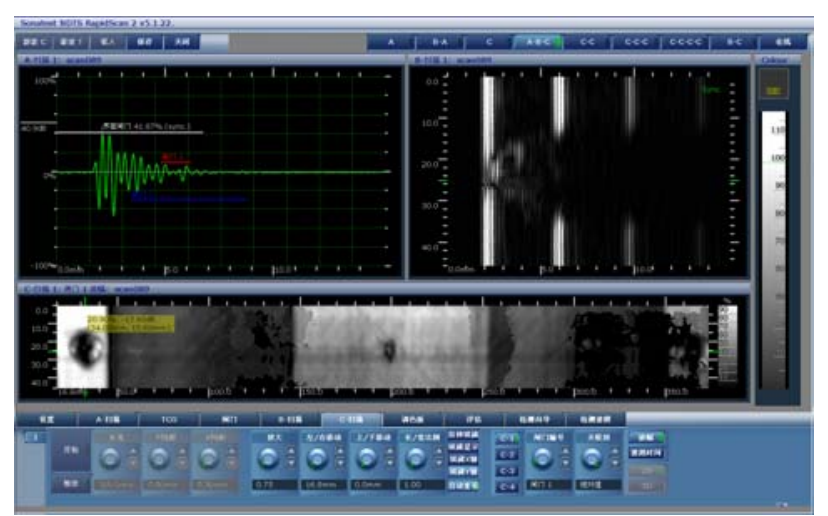

Figure 4. The crater depth of specimen II at the energy of $25 \mathrm{~J}$.

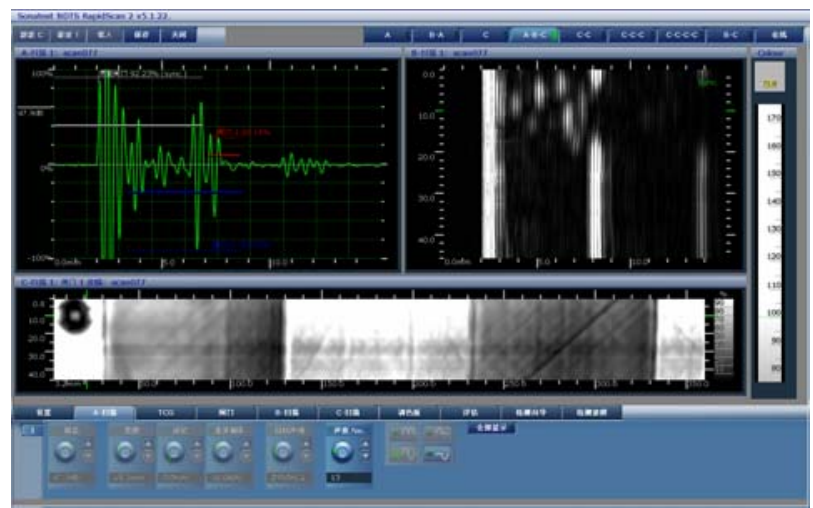

Figure 5. The crater depth of specimen III at the energy of $35 \mathrm{~J}$.

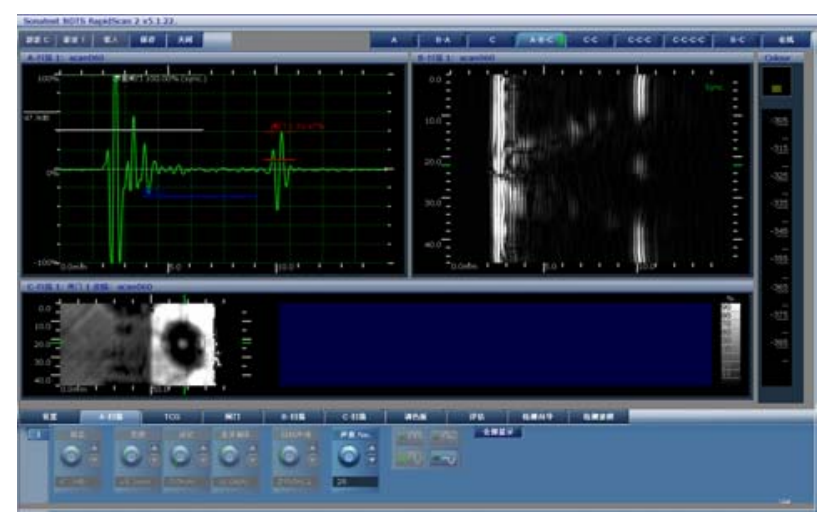

Figure 6. The crater depth of specimen IV at the energy of $45 \mathrm{~J}$.

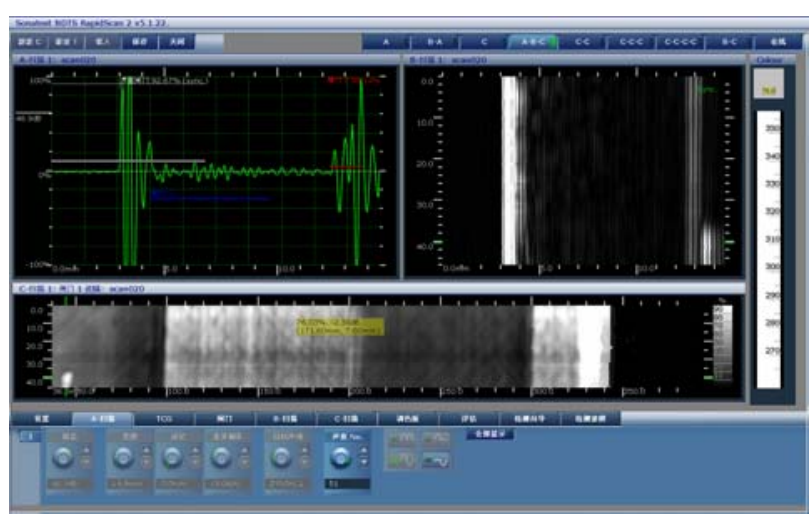

Figure 7. The crater depth of specimen $\mathrm{V}$ at the energy of $45 \mathrm{~J}$.

\subsection{Tensile and leakage test assessment}

Figure 8 shows the impact energy initial value of Nitrogen and aviation kerosene leakage of specimen I to specimen $\mathrm{V}$.

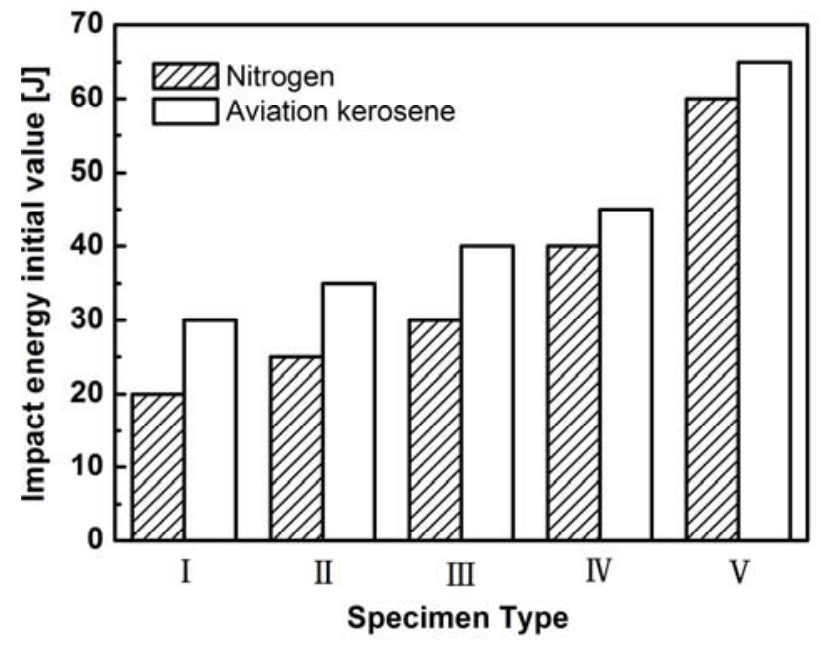

Figure 8. Impact energy initial value of Nitrogen and aviation kerosene leakage.

As shown in Figure 8, the impact energy initial value of Nitrogen and aviation kerosene leakage increases with the increasing thickness of CFRP laminates.

Table 4 shows that the impact energy initial value of Nitrogen leakage is lower than that of aviation kerosene leakage.

Table 4. Impact energy initial value of medium leakage.

\begin{tabular}{|c|c|c|}
\hline \multirow{2}{*}{ Specimen type } & \multicolumn{2}{|c|}{ Impact energy initial value (J) } \\
\cline { 2 - 3 } & Nitrogen & Aviation kerosene \\
\hline I & 20 & 30 \\
\hline II & 25 & 35 \\
\hline III & 30 & 40 \\
\hline IV & 40 & 45 \\
\hline V & 60 & 65 \\
\hline
\end{tabular}


Figure 9 shows that the impact energy initial value of Nitrogen leakage is about 5.8763 times of the thicknesses of laminates. As shown in Figure 4, the impact energy initial value of aviation kerosene leakage is about 6.7416 times of the thicknesses of laminates, which is coordinate with the experience value of 6.75 times.

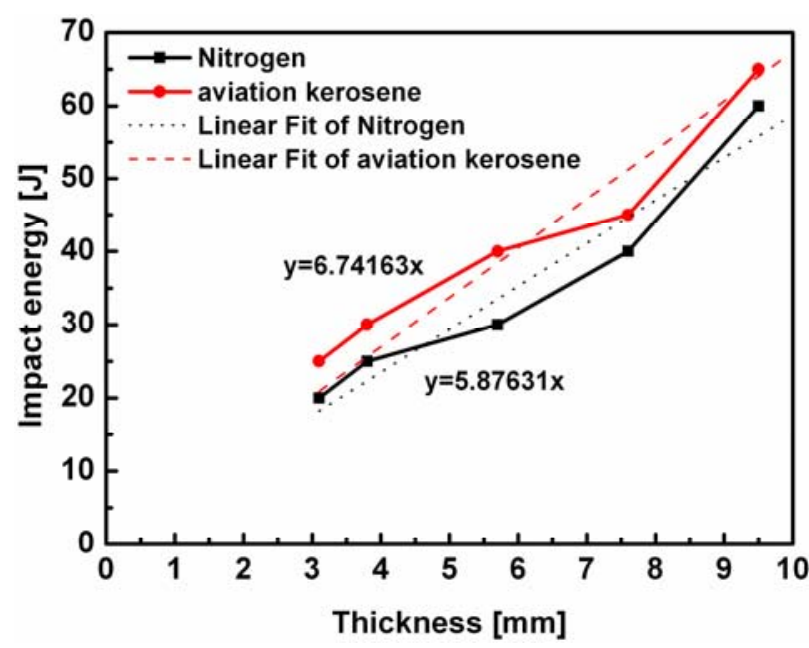

Figure 9. Curves of the impact energy initial value of medium leakage.

\section{Conclusions}

The effects of thickness and impact energy on the impact damage of CFRP laminates are evaluated. Through systematic investigation, three conclusions were drawn.

The crack lengths increase linearly with the increasing impact energy. For the same impact energy, the crack lengths decrease with the increasing specimen thickness.

The impact energy initial value of medium leakage increases with increasing thickness. The impact energy initial value of Nitrogen leakage is lower than that of aviation kerosene leakage.

The impact energy initial value of aviation kerosene leakage is about 6.7416 times of the thicknesses of laminates, which is coordinate with the experience value of 6.75 times.

\section{Acknowledgements}

This work was financially supported by Heilongjiang Natural Science Foundation (Grant No. E201454).

\section{References}

[1] S.A. Baeurle, A. Hotta, A.A. Gusev. Polymer. 47, 6243 (2006)

[2] A.R. Chambers, J.S. Earl, et al. Int J Fatigue. 28, 1389 (2006)

[3] F. Awaja, B. Arhatari, et al. Polym Degrad Stab. 94, 814 (2009)

[4] P.D. Puglia, M.A. Sheikh, D.R. Hayhurstb. Compos Part A. 35, 223 (2004)

[5] B.R. Stoner, S.R. Akshay, et al. Appl Phys Lett. 99 , $183104(2011)$
[6] C.B. Parker, S.R Akshay, et al. J. Mater. Res. 27, $1046(2012)$

[7] O. Asi. Compos Struct. 90 (2009), 43

[8] V. P. W Shim, L. M. Yang. Int J Mech Sci. 47, 647 (2005)

[9] Y. Aoki, K. Yamada, T. Ishikawa. Compos. Sci. Technol. 68, 1376 (2008)

[10] M.F.S.F. Moura, A.T. Marques. J Compos Mater. 19, 1462 (1997)

[11]Z.Y. Zhang, M.O.W. Richardson. Compos Struct. 81, 195 (2007)

[12]G. Minak, P. Morelli, A. Zucchelli. Compos. Sci. Technol. 69, 1358 (2009)

[13]P. N. B. Reis, J. A. M. Ferreira and et al: Compos. Sci. Technol. 60, 154 (2009)

[14]C. Santiuste, S. Sanchez-Saez, and E. Barbero: Compos Struct. 92, 25 (2010)

[15]T. R. Andrew, B. Richard and W. H. Giles: Int J Solids Struct. 45, 4349 (2008)

[16]M. F. S. F. Moura, A. T. Marques. Compos Part A. 33, 361 (2002)

[17]K.W. Kang, H.S. Kim, et al. Mater Sci Eng. A483484, 333 (2008)

[18] M. L. Costa, M. C. Rezende, S. F. M. Almeida. J Compos. Mater. 39, 1943 (2005)

[19]K. H. Im, C. S. Cha, et al. Compos Part B. 32 , 669(2001) 